\title{
Electrode Design for Biochemical Reagent Concentration Measurement
}

\author{
Chan-Young Park, ${ }^{1,2}$ Mi-So Lee, ${ }^{1,2}$ Yu-Seop Kim, ${ }^{1,2}$ \\ Hye-Jeong Song, ${ }^{1,2}$ and Jong-Dae Kim ${ }^{1,2 *}$ \\ ${ }^{1}$ Department of Convergence Software, Hallym University, \\ 1 Hallymdaehak-gil, Chuncheon-si, Gangwon-do 24252, Korea \\ ${ }^{2}$ Bio-IT Research Center, Hallym University, \\ 1 Hallymdaehak-gil, Chuncheon-si, Gangwon-do 24252, Korea
}

(Received May 27, 2018; accepted October 11, 2018)

Keywords: printed circuit board, tapes, plastic film, electrode, impedance spectroscopy, label-free technique

In this paper, we propose a system for estimating the concentration of biochemical reagents through impedance measurement. In particular, we studied electrode structures that can accurately measure impedance. We have fabricated electrodes using printed circuit boards (PCBs), tapes, plastic films, and copper tapes applicable to existing mass production facilities and investigated their performance. When the PCB pattern includes a pair of electrodes, a tape or plastic film is used to make a well to hold the reagent. On the other hand, when two pieces of copper tape were used, a hole in one piece acts as a well. The performances of various electrode structures were compared by obtaining the coefficient of variation in the measurement through repeated measurements of the impedances of various saline solutions of known concentrations. Experimental results show that the impedance of a reagent can be measured stably using copper electrodes.

\section{Introduction}

Most biosensors capable of detecting biomolecules such as nucleic acids, proteins, and cells employ fluorescence labeling techniques for the sensitive and specific detection of biomolecules. ${ }^{(1-4)}$ Label-free techniques have strong advantages over traditional fluorescence labeling techniques, such as simplicity of analysis procedures and reusability of samples. ${ }^{(4-10)}$

Previously, an example of measuring the concentration of a biochemical reagent through impedance measurement by a label-free technique was reported, and electrodes of various materials were made on CMOS, glass, and plastic films. ${ }^{(2,3,10-12)}$ However, since these electrodes do not have a stable mass production facility, the development and stabilization of mass production facilities are essential to realize their practical use.

On the other hand, general-purpose impedance measurement devices such as a lock-in amplifier, a frequency or network analyzer, and LCR meters are being used for the impedance measurement, but they require a lot of space and are difficult to control..$^{(2,7,13-15)}$ Recently, many

*Corresponding author: e-mail: kimjd@hallym.ac.kr https://doi.org/10.18494/SAM.2019.2089 
high-performance USB oscilloscopes, which usually have a built-in function generator, making them suitable for the impedance measurement of biological samples, have been developed. Most of them provide software development kits for the computing platform, so if they are combined with a single-board computer (SBC), space and cost can be reduced significantly. In addition, recently launched SBCs are flexible in their networking capabilities, making it easy to implement systems in an Internet-of-things (IoT) form. ${ }^{(16,17)}$

When the apparatus for measuring the concentration of a biochemical reagent is implemented in IoT form, various advantages other than the basic benefits of the IoT apparatus can be obtained. Using the web-based user interface, one can perform system control and monitoring without standard input/output devices such as the monitor or keyboard/mouse, so space for input/output devices can be reduced. It also has the advantage of enabling monitoring and discussion of experimental situations with colleagues anytime, anywhere using Internetconnected standard computing devices such as personal computers or smart devices. ${ }^{(18)}$

In this paper, we propose a reagent impedance measurement system in IoT form, and investigate the structure of electrodes that are connected to the proposed system and provide stable measurement. Wells for reagent containment and electrodes were constructed using printed circuit boards (PCBs), copper tapes, plastic tapes, and films produced in well-developed mass production facilities. To compare the various electrodes, the coefficient of variation (CV) was obtained by repeatedly measuring the impedance of a saline solution at various concentrations. Experimental results showed that the copper tape electrode enables the stable measurement of the impedance of the saline solution.

\section{Materials and Methods}

Figure 1 shows the IoT-type biochemical reagent measurement system proposed in this paper. The sine wave generated from the function generator of the USB oscilloscope (Analog Discovery 2, Digilent, USA) adopted in this system is applied to the electrode through the amplifier. The impedance of the solution exposed to the electrodes can be determined from the applied voltage (chA), the amplifier output (chB), and the reference resistance R measured by the oscilloscope. In the inverting amplifier structure shown in the figure, the gain of the amplifier is easily obtained because it is expressed by the ratio of the reference resistance to the solution impedance. The oscilloscope is connected to the SBC and controlled by the

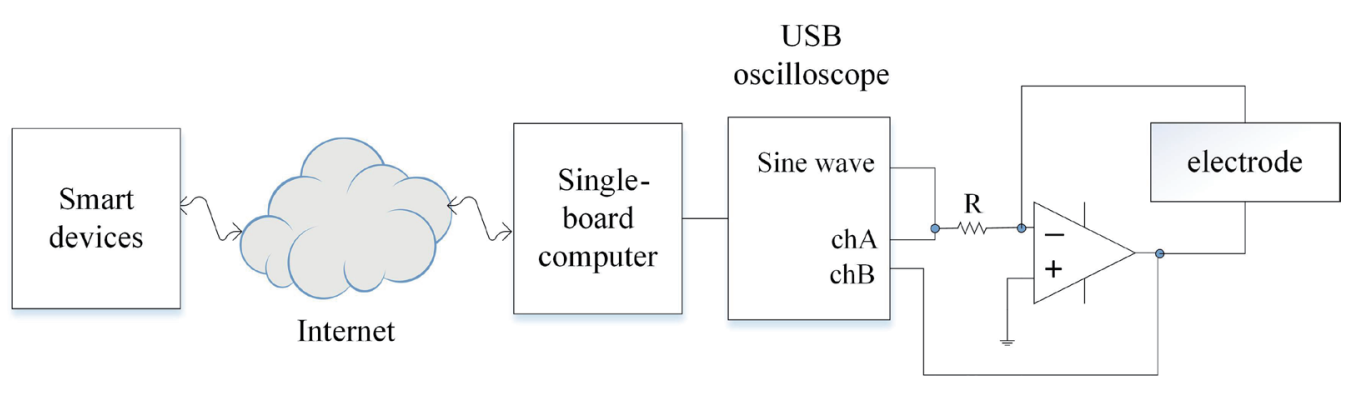

Fig. 1. (Color online) Proposed system. 


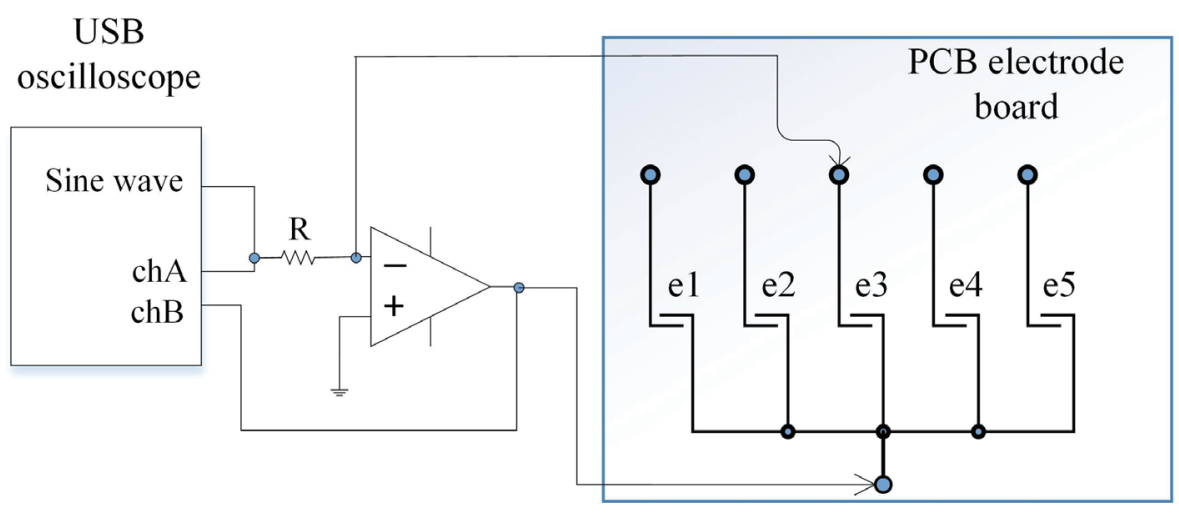

Fig. 2. (Color online) Example of connection example for one of the PCB electrode pairs.

Python program, which is written with the software development kit provided by the USB oscilloscope supplier. The SBC has the Jupyter notebook server that can be accessed through any smart device connected to the Internet. The user can access the Python control program via the server. Figure 2 shows an example of a connection between the oscilloscope and one of the electrode pairs of the PCB with 5 electrode pairs. When forming a pair of electrodes with copper tape, we made only a pair of electrodes because we had to make all the holes and tapes manually.

In this experiment, a $10 \mathrm{kHz}, 1 \mathrm{~V}$ peak-to-peak sine wave is generated and applied to the electrode through a resistance of $100 \mathrm{k} \Omega$. Five identical electrodes were made on a PCB to measure the interdigitated electrode variation in the case of the PCB electrode. The oscilloscope adopted in this study provides a $\pm 5 \mathrm{~V}$ power supply in addition to the function generator, which is used to generate the bias voltages of the amplifier. The PCB electrodes tested in this work were of four kinds with interdigitated electrodes with 1 to 4 fingers and only one pair of electrodes was constructed using copper tapes. However, in the case of PCB electrodes, it is advantageous for the electrodes to be small in order to minimize the amount of reagent to be measured and to reduce the fluctuation in the experiment. Therefore, only 1-finger electrodes were selected and tested. Figure 3 is a photograph of the 1-finger electrode PCB used in the experiment. To accurately measure a small amount of solution, the shape of the well is critical rather than the structure of the electrode. Therefore, wells were constructed using various tapes and films. Among them, the results for two types of wells showing meaningful results are presented in this paper along with the cases without a well. In this paper, we show experimental results for four types of electrode configurations as a whole; three of them have various wells on the electrode PCB and the fourth is the copper tape electrode pair. The three types of PCB electrodes were those without wells, those with holes and polycarbonate film, and those with holes and a box tape. Five concentrations of saline solution, i.e., 10, 5, 2.5, 1.25, and 0.625 $\mathrm{mg} / \mathrm{ml}$, were prepared for comparing the various electrodes.

First, the PCB electrode pairs without wells were tested. One microliter of the prepared solution was applied dropwise to uniformly cover each electrode pair on the PCB. After confirming under a microscope that the reagent uniformly covered the electrode pair, impedance was measured using the proposed impedance measurement system. This procedure 


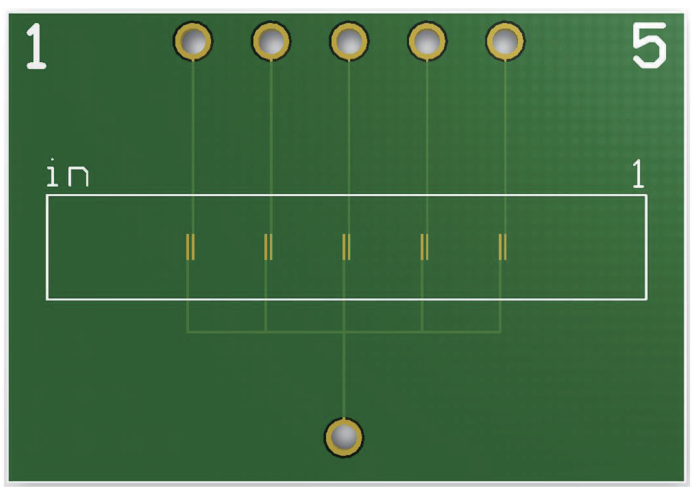

Fig. 3. (Color online) 1-finger interdigitated electrode PCB.

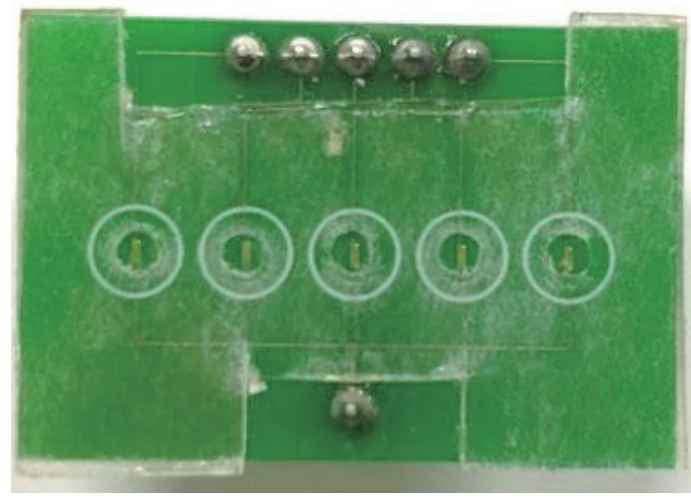

Fig. 4. (Color online) Electrode with polycarbonate tape with wells.

was repeated 10 times at all concentrations of reagent. This method can result in imprecise impedance measurements because the location where the reagent dropped on the electrode is irregular and the reagent is unevenly distributed.

To prevent an uneven drop, we prepared holes as the wells in the polycarbonate film, as shown in Fig. 4, by attaching a double-sided tape to a $500-\mu \mathrm{m}$-thick polycarbonate film and then drilling holes of $2 \mathrm{~mm}$ diameter to match the positions of the electrodes on the PCB chip. When a film or tape with wells was attached, the reagent could leak between the tape and the PCB. Therefore, after attaching the tape with wells to the chip, the chip was laminated at a temperature of $60{ }^{\circ} \mathrm{C}$ for $1 \mathrm{~s}$ using a laminator (ProLami DCL-3306D, Copierland, Korea). The completed PCB chip was tested by the first method. In this case, the amount of reagent and the area of the reagent contacting the PCB chip were the same, so that the impedance could be measured uniformly. However, air bubbles generated when dropping the reagent into the wells interfered with the contact between the electrode and the reagent, resulting in a large error. Also, we found that, as the experiment was repeated, the reagent leaked and the measured impedance tended to become higher. To prevent this, we increased the number of laminations, but it did not help.

A third configuration was tried to solve this problem. The material of the chamber was changed from polycarbonate to a $65 \mu \mathrm{m}$ oriented polypropylene (OPP) tape (Fig. 5). Holes of 2 $\mathrm{mm}$ diameter were drilled on the tape to align the positions of electrodes on the PCB chip. With the OPP tape, the height of the wells was very small compared with the amount of reagent, so the generation of air bubbles was minimized. However, similar to the second configuration, the more the experiment was repeated, the more the reagent leaked between the PCB chip and the tape.

When the entire electrode is made of metal, its hydrophilic nature attracts the reagent to the entire electrode. This property can help even application of the reagent over the electrode. To take advantage of this property, electrodes were made using copper tape, as shown in Fig. 6. After attaching a double-sided tape to a copper tape, a hole of $2 \mathrm{~mm}$ diameter was drilled and this tape was attached to a nonperforated copper tape. Then, lamination was performed with the same settings as before. In this way, the electrode and the well were realized simultaneously. 


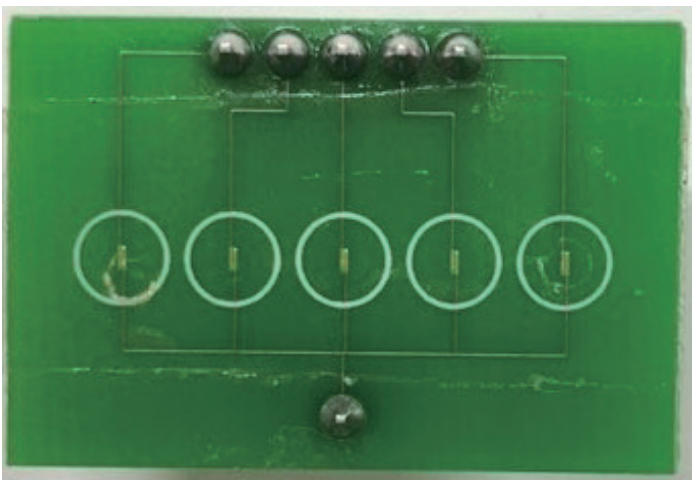

Fig. 5. (Color online) Electrode with OPP tape with wells.

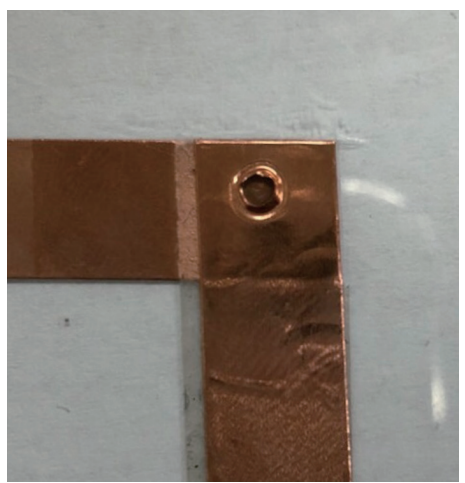

Fig. 6. (Color online) Electrode constructed using copper tape.

\section{Result}

To compare the accuracies of the methods, we measured the concentration of each reagent 10 times and then compared the CVs. Table 1 shows the results of the PCB electrode test without wells. The electrode pairs are numbered sequentially from the left in Fig. 3. Since no well was constructed on the electrode, the reagent often did not cover the electrode uniformly (Fig. 7), and the measured impedance varied greatly from 5.1 to $24.8 \%$.

To compensate for the above disadvantages, a $500-\mu \mathrm{m}$-thick polycarbonate tape with wells was constructed with the expectation that the reagent would be evenly distributed on the electrode pair. However, since the height of the well was greater than the height of the reagent, air bubbles were easily formed on the electrode when the reagent was dropped (Fig. 8). Therefore, the CVs were smaller than those on the electrode without a well, but not satisfactory $(2-117.9 \%)$. This problem was not resolved by increasing the amount of reagent to $2 \mu 1$. Table 2 shows the CV for the PCB electrode test using the polycarbonate tape with wells.

Even when OPP was used for forming the well, it was difficult to drop the reagent evenly as in the case of the well-free structure, so the $\mathrm{CV}$ of the measured impedance was higher than that in the case of the polycarbonate tape with wells. Therefore, the CV results of the OPP well experiment were not included in this paper. Figure 9 shows an example of an irregular drop over the OPP tape with wells.

In the case of a copper tape electrode, the reagent could be uniformly applied to the electrode pair owing to the hydrophilicity of the metal. Since this structure showed highly satisfactory results, we tested whether the same performance could be obtained over several days. Table 3 shows the CVs of the electrode test using the copper tape electrode for 3 days. The second experiment was carried out the day after the first experiment, and the third experiment was carried out three days after the second experiment. Measurements were very stable in the first two experiments (CVs less than 2.8\%). However, in the third trial, the CVs increased on average. Microscopy examination showed that there were parts that were lifted under the double-sided tape as a result of repeatedly wiping off the reagent. The results showed that the proposed copper tape electrodes can reliably measure the impedance of the reagent if this problem can be resolved or the device is used as a disposable one. 
Table 1

Coefficients of variation of measured impedance for the structure without a well (\%).

\begin{tabular}{lccccc}
\hline Electrode pair ID & 1 & 2 & 3 & 4 & 5 \\
\hline $0.625 \mathrm{mg} / \mathrm{ml}$ & 5.1 & 6 & 8.3 & 7.3 & 7.7 \\
$1.25 \mathrm{mg} / \mathrm{ml}$ & 4.9 & 10.8 & 13.1 & 9.6 & 8.3 \\
$2.5 \mathrm{mg} / \mathrm{ml}$ & 15.4 & 8.6 & 11.6 & 13.2 & 11.4 \\
$5 \mathrm{mg} / \mathrm{ml}$ & 17.1 & 21.6 & 19.4 & 24.8 & 13 \\
$10 \mathrm{mg} / \mathrm{ml}$ & 7.5 & 12.1 & 9 & 9.3 & 15.7 \\
\hline
\end{tabular}

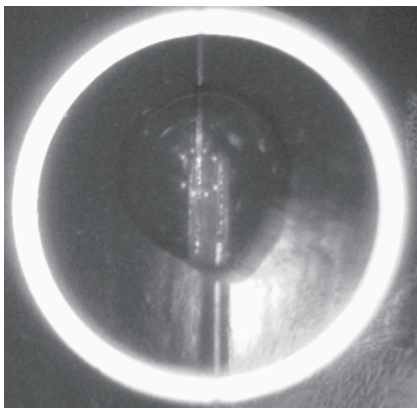

Fig. 7. Uneven drop for no-well construction.

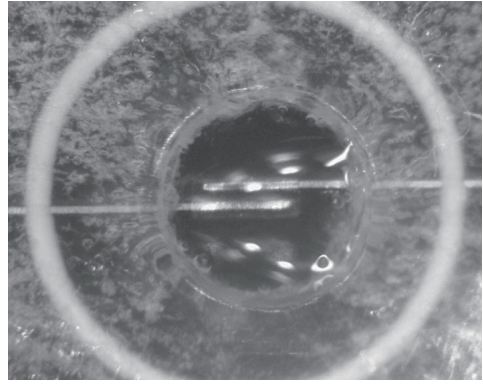

Fig. 8. Air bubbles in the polycarbonate well.

Table 2

Coefficients of variation of measured impedance for the structure with the polycarbonate tape with wells (\%).

\begin{tabular}{lrrrll}
\hline Electrode pair ID & 1 & \multicolumn{1}{c}{2} & \multicolumn{1}{c}{3} & 4 & 5 \\
\hline $0.625 \mathrm{mg} / \mathrm{ml}$ & 7.7 & 10.2 & 10.2 & 3.3 & 4.1 \\
$1.25 \mathrm{mg} / \mathrm{ml}$ & 9.2 & 7.1 & 117.9 & 2 & 1.3 \\
$2.5 \mathrm{mg} / \mathrm{ml}$ & 10.6 & 7.3 & 15.8 & 2.9 & 9.2 \\
$5 \mathrm{mg} / \mathrm{ml}$ & 8.5 & 9.3 & 8.6 & 7.6 & 2.7 \\
$10 \mathrm{mg} / \mathrm{ml}$ & 5.6 & 5.5 & 4.7 & 4.9 & 10.1 \\
\hline
\end{tabular}

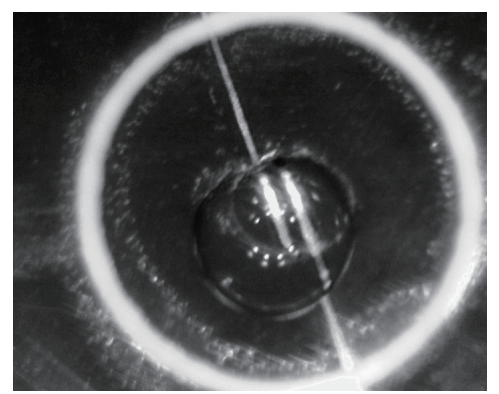

Table 3

Coefficients of variation of copper electrode.

\begin{tabular}{lccr}
\hline Measurement day & 1st & 2nd & 3rd \\
\hline $0.625 \mathrm{mg} / \mathrm{ml}$ & 2.8 & 1.2 & 11.8 \\
$1.25 \mathrm{mg} / \mathrm{ml}$ & 1.5 & 0.9 & 1.3 \\
$2.5 \mathrm{mg} / \mathrm{ml}$ & 2.4 & 2.1 & 1.9 \\
$5 \mathrm{mg} / \mathrm{ml}$ & 1.8 & 1.5 & 26.7 \\
$10 \mathrm{mg} / \mathrm{ml}$ & 1.1 & 1.4 & 4.3
\end{tabular}

Fig. 9. Uneven drop on the OPP well.

\section{Conclusions}

In this paper, we proposed an IoT-type reagent measurement system and investigated electrodes that can be connected to this system to measure the impedance of a reagent stably. The analog front end was configured with a USB oscilloscope and a simple amplifier and controlled by a SBC with a built-in Jupyter notebook server. The proposed system can be 
controlled and observed using any smart device connected to the Internet. Among the various electrodes investigated in this paper, the proposed copper tape electrode stably measured impedance with CVs of less than $2.8 \%$.

\section{Acknowledgments}

This research was supported by the Basic Science Research Program through the National Research Foundation of Korea (NRF) funded by the Ministry of Science, ICT \& Future Planning (NRF-2017R1A2B4002679) and by The Leading Human Resource Training Program of Regional Neo Industry through the National Research Foundation of Korea (NRF) funded by the Ministry of Science, ICT \& Future Planning (NRF-2016H1D5A1909654).

\section{References}

1 R. E. Cunningham: Fluorescent Labeling of DNA: Immunocytochemical Methods and Protocols (Springer, Totowa, 1999) p. 271.

2 H. Ma, R. W. Wallbank, R. Chaji, J. Li, Y. Suzuki, C. Jiggins, and A. Nathan: Sci. Rep. 3 (2013) 2730.

3 C.-M. Chen and M. S.-C. Lu: 19th Int. Conf. Solid-State Sensors, Actuators and Microsystems (TRANSDUCERS) (2017) 1632.

4 I. O. K'Owino and O. A. Sadik: Electroanalysis 17 (2005) 2101.

5 D. Holmes, D. Pettigrew, C. H. Reccius, J. D. Gwyer, C. van Berkel, J. Holloway, D. E. Davies, and H. Morgan: Lab Chip 9 (2009) 2881.

6 R. McGuinness: Curr. Opin. Pharmacol. 7 (2007) 535.

7 P.-D. Nguyen, T. B. Tran, D. T. X. Nguyen, and J. Min: Sens. Actuators, B 197 (2014) 314.

8 R. Sharma, S. E. Deacon, D. Nowak, S. E. George, M. P. Szymonik, A. A. Tang, D. C. Tomlinson, A. G. Davies, M. J. McPherson, and C. Walti: Biosens. Bioelectron. 80 (2016) 607.

9 Y. Hu, K. Wang, Q. Zhang, F. Li, T. Wu, and L. Niu: Biomaterials 33 (2012) 1097.

10 A. Manickam, A. Chevalier, M. McDermott, A. D. Ellington, and A. Hassibi: 2010 IEEE International SolidState Circuits Conf. Digest of Technical Papers (ISSCC) (IEEE, 2010) 130.

11 A. S. Mucha: Doctoral Thesis, Technical University of Munich (2012).

12 M. Shariati: Biosens. Bioelectron. 105 (2018) 58.

13 R. Sinville and S. A. Soper: J. Sep. Sci. 30 (2007) 1714.

14 Z. Zou, S. Lee, and C. H. Ahn: IEEE Sens. J. 8 (2008) 527.

15 T. Schwarzenberger, P. Wolf, M. Brischwein, R. Kleinhans, F. Demmel, A. Lechner, B. Becker, and B. Wolf: Physiol. Meas. 32 (2011) 977.

16 S. Ferdoush and X. Li: Procedia Comput. Sci. 34 (2014) 103.

17 J. Miranda, J. Cabral, S. R. Wagner, C. F. Pedersen, B. Ravelo, M. Memon, and M. Mathiesen: Sensors 16 (2016) 2089.

18 I. Lee and K. Lee: Bus. Horiz. 58 (2015) 431. 